\title{
PARASITISMO GASTRINTESTINAL E HEMATOLOGIA EM EQUINOS E ASININOS DA MESORREGIÃO DA AGLOMERAÇÃO URBANA, SÃO LUÍS, MARANHÃO
}

\author{
George Montalvane Silva Ferreira ${ }^{1}$, Francisca Andreia Ferreira Dutra, ${ }^{1}$ Edvaldo \\ Franco Amorim Filho ${ }^{1}$, Ana Clara Gomes dos Santos ${ }^{1}$ \\ 1 UEMA \\ Correspondência: George Montalvane Silva Ferreira: george.mont@hotmail.com
}

RESUMO: Objetivou-se avaliar as alterações hematológicas relacionadas à carga parasitária gastrintestinal em equinos e asininos naturalmente infectados na llha de São Luis, MA. Foram selecionados aleatoriamente 57 equinos e 37 asininos adultos, sem raça definida, de idade e pesos variados. Exames coproparasitológicos foram realizados pelos métodos de Willis-Mollay, GordonWhitlock, Robert O'Sulivan. Os animais foram distribuídos em quatro grupos, conforme carga parasitária e espécie animal em: grupo 1 - asininos com OPG $\leq 500$; grupo 2 - asininos com OPG $>500$; grupo 3 - equinos com OPG $\leq 500$; e grupo 4 - equinos com $O P G>500$. Os resultados obtidos na contagem de ovos por gramas de fezes (OPG) apresentaram uma variação de 0 a 3.000 OPG para equinos e 0 a 6.900 para asininos, a pesquisa de larvas em ambas as espécies indicou maior frequência para a associação Cyathostominae - Strongylus vulgaris, o que determinou principalmente no grupo dos asininos com OPG $\leq 500$, valores médios elevados na contagem total de leucócitos, bastonetes, eosinófilos e linfócitos. Tais achados apontam a existência de leve leucocitose com eosinofilia associada ou não a elevação de outras células, demonstrando que a eosinofilia foi um sinalizador de presença de parasitismo intestinal para asininos, mesmo quando estes apresentam valores baixos para a contagem do OPG.

Palavras-chave: equideos; Strongylus vulgaris; valores leucocitários

\section{GASTROINTESTINAL PARASITISM AND HEMATOLOGY IN HORSES AND DONKEYS FROM THE URBAN AREA OF SÃO LUÍS/MA, NORTHEAST BRAZIL}

ABSTRACT: This research paper aimed to evaluate the hematological changes related to natural gastrointestinal parasite load in infected horses and donkeys on the island of Sao Luis, MA. The animals selected were 57 horses and 37 donkeys, mixed breed, varied ages and weight, were randomly selected. Fecal examinations were performed by Willis-Mollay, Gordon-Whitlock, and Robert O'Sulivan methods. The animals were divided into four groups according to parasitic load and animal species in: group 1 - donkeys with EPG $\leq 500$; group 2 - donkeys with EPG > 500; group 3 horses with $E P G \leq 500$, and group 4 - horses with $E P G>500$. The results obtained in eggs per gram of feces counting (EPG) showed a variation from 0 to 3,000 EPG for horses and 0 to 6,900 for donkeys; the larval culture results in both species showed a higher frequency of Cyathostominae Strongylus vulgaris association which resulted, mainly in the group of donkeys with $\leq 500$ EPG, high average values in leukocyte, rod cells, eosinophils, and lymphocytes total counting. Such findings indicate the existence of mild leukocytosis with eosinophilia associated or not with the elevation of other cells, showing that eosinophilia was a marker for the presence of intestinal parasitism for donkeys, even when they have low values of EPG counting.

Key Words: equines; Strongylus vulgaris; leukocytes values 


\section{INTRODUÇÃO}

Os equinos (Equus caballus) e asininos (Equus asini) são hospedeiros de grande variedade de helmintos, principalmente os nematóides pertencentes à Superfamília Strongyloidea (Strongylus vulgaris, Strongylus edentatus, Ciatostomíneos, Trichostrongylus axel), Ascarioidea (Parascaris equorum), Rhabdiasoidea (Strongyloides westeri), Oxyuriodea (Oxyuris equi) e cestódeos da Família Anaplocephalidae (Anoplocephala perfoliata) (Lichtenfels, 1975).

Apesar de serem mais resistentes que os cavalos, os asininos, como os demais equídeos apresentam uma grande variedade de parasitos em sua fauna helmíntica. As formas de criação destes animais, bem como seus hábitos alimentares favorecem a grande incidência de infecções parasitárias (Molento, 2005).

A forma larval no parasitismo gastrintestinal causa uma reação inflamatória, caracterizada por influxo de neutrófilos, eosinófilos, e macrófagos, ao penetrar na mucosa intestinal. Devido à migração das larvas ocorre arterite ileocecocólica evidenciada histologicamente por inflamação multifocal, necrose e fibrose, havendo evidências de que o fator de necrose tumoral (TNF) e a interleucina (IL-1) sejam os mediadores-chave envolvidos em deflagrar a cascata inflamatória (Drudge et al. 1966, Duncan \& Pirie 1975, Drudge 1979, Slocomb 1985, Klei et al. 1990, Morris 1991, Moore et al. 1995).

A fauna parasitária helmíntica em equinos e asininos é ampla e de relevante importância para a sanidade animal, expressa através da carga parasitária em ovos por gramas de fezes (OPG), podendo desencadear alterações sistêmicas graves associadas ao parasitismo gastrintestinal, que podem ser diagnosticadas nos exames hematológicos. Dessa forma considerando a inexistência de pesquisas realizadas sobre helmintos gastrintestinais, carga parasitária, identificação larval associada às alterações hematológicas nessas espécies de animais, se fez necessária a realização dessa pesquisa.

\section{MATERIAL E MÉTODOS}

A pesquisa foi realizada na llha de São Luís localizada pelas coordenadas geográficas; $2^{\circ} 24^{\prime} 10^{\prime \prime}$ e $2^{\circ} 46^{\prime} 37^{\prime \prime}$ de latitude Sul e $44^{\circ} 22^{\prime} 39^{\prime \prime}$ e $44^{\circ} 22^{\prime} 39^{\prime \prime}$ de longitude Oeste, com área de $31,7 \mathrm{~km}^{2}$, no litoral Norte do Estado do Maranhão, Costa Setentrional do Brasil (NugelUema 2008, lbge 2002).

$\mathrm{Na}$ pesquisa foram utilizados 57 equinos e 37 asininos adultos, naturalmente infectados por helmintos, sem raça definida (SRD), com idade e peso variados, independentes de sexo. Estes animais foram distribuídos em quatro grupos em função da carga parasitária $(\mathrm{CP})$ sendo o ponto de corte do OPG (ovos por gramas de fezes) no valor de 500 e de acordo com a espécie animal em: Grupo 1 - asininos com OPG $\leq 500$; Grupo 2 - asininos com OPG $>500$; Grupo 3 - equinos com OPG $\leq 500$; e Grupo 4 - equinos com OPG $>500$, comparações das variáveis hematológicas (leucócito total, diferencial e hematócrito), LPG (larvas por gramas de fezes) e a associação parasitária por diferentes gêneros de helmintos.

Foi realizada a contenção física de cada animal, para a coleta das amostras de fezes diretamente da ampola retal, com auxílio de luvas de inseminação, identificadas individualmente, acondicionadas em gelo e conduzidas ao Laboratório de Parasitologia da Universidade Estadual do Maranhão UEMA. 
aglomeração urbana, São Luís, Maranhão

As amostras de sangue foram colhidas por venopunção da jugular cervical em frascos contendo ácido etilenodiaminotetracético, sal dissódico (EDTA) na proporção de 1 gota/5mL de sangue total, no período da manhã, sendo conduzidos para análise ao Laboratório de Patologia Clínica/UEMA. As variáveis hematológicas foram obtidas conforme os métodos clássicos preconizados por Jain (1986).

Os métodos coproparasitológicos utilizados foram: a técnica modificada de Gordon \& Whitlock (1939), Roberts \& O'Sulivan (1950), e a identificação de larvas de helmintos gastrintestinais, de acordo com Hoffmann (1987).

A contagem de leucócitos totais foi realizada em câmara de Neubauer, de acordo com as recomendações de Jain (1993) e Meyer \& Harvey (2004); enquanto, a contagem diferencial de leucócitos foi realizada em esfregaços sanguíneos simples em lâminas de microscopia corados pelo método hematológico rápido (Panótico), para obtenção dos valores percentuais e absolutos de cada tipo celular específico (neutrófilos, bastonetes, linfócitos, monócitos, eosinófilos, basófilos).

A determinação do volume globular (hematócrito) foi realizada por meio da técnica de microcentrifugação com o auxílio de tubos capilares na velocidade de 3.500 a 5.000 rpm durante 5 min., a leitura foi realizada segundo a técnica de Colles (1984).

$\mathrm{Na}$ Análise Estatística as médias da CP (OPG) foram analisadas pela Análise de Variância (ANOVA) seguida pelo teste de Tukey-Kramer, sendo fixada a probabilidade de erro tipo $1 \mathrm{em}$ 10\% (Serra-Freire 2002).

\section{RESULTADOS E DISCUSSÃO}

Nos resultados observados quanto ao parasitismo em equinos e asininos verificou-se carga parasitária de leve a intensa, assim como ausência e elevada concentração de ovos, isto é, acima de 23 por campo microscópico, principalmente em asininos, sem presença de sintomas aparentes indicativos de nematodiose. Em equinos foram observados alguns casos isolados de cólica e diarréia apesar dos animais com OPG nulo e abaixo de 500 terem sido predominantes. Os equinos apresentaram maior frequência de animais parasitados $84,22 \%$ para (CP $\leq 500$ OPG) e $15,78 \%$ (CP >500 OPG), observou-se que a maioria dos animais $(77,19 \%)$ estava com parasitismo leve com $0 \leq \mathrm{OPG} \leq 300$, dos 57 animais examinados. Nos asininos foram verificados percentuais de $72,97 \%$ (CP $\leq 500$ OPG) e $27,03 \%$ (CP >500 OPG) dos 37 animais examinados. $A$ frequência de parasitismo entre espécies animais foi de $15,78 \%$ para equinos e $27,03 \%$ para asininos com (CP >500 OPG). Dessa forma, os asininos apresentaram maior grau de parasitismo quando comparados aos equinos (Tabela 1 ).

TABELA 1 - Comparação de carga parasitária (OPG) de helmintos gastrintestinais da Superfamilia Strongyloidea, entre equinos e asininos naturalmente infectados na llha de São Luís, MA, periodo de 2008 a 2009.

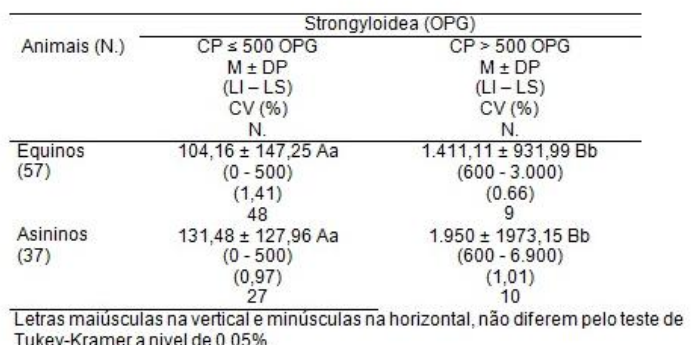

Nos exames parasitológicos realizados foram verificados ovos da Superfamília Strongyloidea (S. vulgaris, $S$ edentatus, Ciatostomíneos), Ascarioidea ( $P$. equorum) e Rhabdiasoidea ( $S$. westeri) para equinos. $E$, para os asininos: Strongyloidea (S. vulgaris, S. edentatus, T. axei, Ciatostomíneos), Rhabdiasoidea (S. westeri), Oxyuroidea (O. equi), Ascarioidea ( $P$. equorum) e cestódeos da Família Anoplocephalidae ( $A$. perfoliata). 
O OPG baixo e mesmo nulo encontrado no grupo dos equinos e asininos com CP ( $\leq 500$ OPG) não descarta a presença de parasitose e alterações sistêmicas, fato este, já registrado em trabalho realizado por Lind et al. (2003) na Suécia, que verificaram em 27 equinos, com $O P G \geq$ 200 ovos, na investigação da composição das espécies de Ciatostomíneos. As seis espécies mais prevalentes foram Cylicocyclus nassatus, Cyathostomum catinatum, Cylicostephanus Cylicocyclus Cylicostephanus Cylicostephanus longibursatus, leptostomus, Correspondendo a $91 \%$ do total de espécies de Ciatostomíneos encontrados, em animais aparentemente sadios.

Os asininos do grupo com CP (>500 OPG) apresentaram valores na variação entre 600 - 6.900 OPG, estes resultados diferem dos valores encontrados por Carvalho et al. (2007), em Portugal, que avaliando o grau de parasitismo em asininos estabulados encontraram níveis de OPG de estrongilídeos, que variaram entre 200 e 2.000 OPG.

Os asininos com CP (>500 OPG) não apresentaram nenhum sinal clínico indicativo de parasitismo e nem atividade física diminuída (animais de tração), fato este já observado por Getachew et al. (2010) que ao estudarem a composição da população gastrintestinal de helmintos em asininos utilizados em tração, na Etiópia, os exames revelaram percentuais de estrongilídeos de $99 \%$, S. westeri $11 \%$, cestódeos $8 \%$ e 0 . equi $2 \%$, sendo que, mais de $55 \%$ desses asininos tinham CP superior a 1.000 OPG e relataram também que a frequência de níveis elevados de carga parasitária com poliparasitismo é comum, sem prejuízo evidente em suas atividades de trabalho físico.
As larvas identificadas foram das Famílias Strongylidae ( $S$. vulgaris, $S$. edentatus) e Trichostongylidae (T. axei), e da Subfamília Cyathostominae. Os equinos com CP $(\leq 500 \quad$ OPG $)$ apresentaram poliparasitismo com prevalência de larvas (LPG) para as espécies de $S$. vulgaris $(56 \%), S$. edentatus $(9 \%)$ e Cyathostominae (35\%); e para a CP (>500 OPG) foi observado larvas de $S$. vulgaris (66\%), $S$. edentatus (28\%) e Cyathostominae (6\%), com isso foi verificado que o maior parasitismo foi por $S$. vulgaris em ambos os grupos estudados (Tabela 2 ).

TABELA 2 - Valores percentuais e absolutos de LPG de helmintos da Familia Strongylidae e Trichostrongylidae em equinos com $\mathrm{CP}(\leq 500 \mathrm{OPG}=5.000 \mathrm{e} \mathrm{CP}>500 \mathrm{OPG}=12.700) \mathrm{e}$ asininos $\mathrm{CP}(\leq 500 \mathrm{OPG}=3.550 \mathrm{e} \mathrm{CP}>500 \mathrm{OPG}=19.500)$, naturalmente infectados por helmintos gastrintestinais na Illha de São Luis, MA, no periodo de 2008 a 2009.

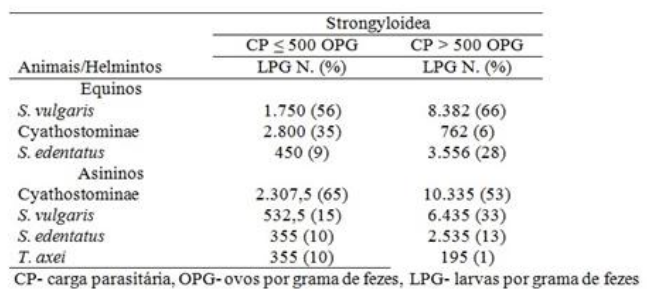

Os equinos apresentaram uma associação entre os helmintos gastrintestinais com CP $(\leq 500$ OPG), entre $S$. vulgaris e Cyathostominae e aqueles com CP (>500 OPG) houve associação entre $S$. vulgaris e $S$. edentatus. Esses resultados estão inferiores aos encontrados por Pereira \& Viana (2006) em 16 cavalos e quatro mulas, que apresentaram parasitismos por Cyathostominae, $S$. vulgaris e $S$. edentatus, com prevalência de 100\%; $70 \%$ e $45 \%$, respectivamente, no entanto são compatíveis quando se observou a associação entre Cyathostominae e $S$. vulgaris, sendo os mais frequentes nesses animais pesquisados.

Os asininos com CP ( $\leq 500$ OPG) também apresentaram poliparasitismo com prevalência de larvas (LPG) em ordem decrescente para Cyathostominae $(65 \%)$ e as espécies de S. vulgaris $(15 \%)$, S. edentatus $(10 \%)$ e T. axei (10\%). E, para a CP (>500 OPG) 
Parasitismo gastrintestinal e hematologia em equinos e asininos da mesorregião da aglomeração urbana, São Luís, Maranhão

foram observadas larvas de Cyathostominae (53\%) e as espécies de S. vulgaris $(33 \%)$, S. edentatus $(13 \%)$ e T. axei (1\%) (Tabela 2). Nos asininos, em ambas as CPs ( $\leq 500$ OPG e $>500$ OPG), foram observadas que as larvas de Cyathostominae apresentaram associação parasitária com $0 \quad S$. vulgaris, e maior frequência em relação às larvas dos grandes estrôngilos $(S$. vulgaris e $S$. edentatus), demonstrando assim que os asininos são mais resistentes a esses parasitos, visto que, não foi observado nenhum sinal clínico indicativo de nematodiose. Ademais, ressalta-se a presença de parasitismo por T. axei, $P$. equorum e o cestódeo $A$. perfoliata nesses animais, no município de São Luís, MA, evento também observado por Pereira \& Viana (2006) que encontraram $85 \%$ de $A$. perfoliata em cavalos e mulas no Vale Paraíba no Estado de São Paulo, Brasil.

Estudos realizados na região de Konya, Turquia, em cavalos e burros de diferentes idades apresentaram prevalência para Strongylidae de 100\%; sendo que os espécimes de $S$. vulgaris e $S$. edentatus foram de; $31,53 \%$ e $17,11 \%$ (cavalos) e, $23,45 \%$ e $14,81 \%$ (burros), respectivamente (Uslu \& Guçlu 2007). Entretanto, esses dados foram superiores aos observados nesse estudo em equinos e asininos, da ilha de São Luís, MA, em virtude de fatores prováveis como: a diferença geográfica entre as regiões, manejo de animais, status fisiológico e sanidade animal que pode proporcionar uma elevada e/ou baixa carga parasitária.

A elevada incidência de Cyathostominae em animais adultos neste trabalho não são compatíveis com os resultados obtidos por Barbosa et al. (2001), que pesquisaram 14 equinos naturalmente infectados por nematóides, oriundos de haras do município de Jaboticabal, São Paulo, Brasil. De um total de 537.128 nematóides recuperados dos 14 animais necropsiados, $80,5 \%$ pertenciam à Subfamília Cyathostominae. Destes, $72,64 \%$ foram encontrados nos equinos jovens e os $27,36 \%$ restantes nos animais adultos. $O$ mesmo registro foi verificado por Umur \& Açiçi (2009) na região central do Mar Negro, Turquia, em cavalos, burros e mulas que mostrou taxas de infecção por Cyathostominae de 33,88\%; 63,79\% e 69,07\%, respectivamente, S. edentatus (31,05\%; $8,62 \%$ e $5,15 \%)$, S. vulgaris $(3,52 \%$; $3,01 \%$ e $8,76 \%$ ), discordando dos registrados nesse estudo que encontraram baixos percentuais em relação ao $S$. vulgaris em equinos e asininos e Cyathostominae em equinos.

$\mathrm{Na}$ Tabela 3, são demonstradas as alterações hematológicas em equinos com CP (>500 OPG) sendo observada leucocitose com neutrofilia, sem desvio à esquerda em dois animais (3,5\%). $E$, para os equinos com CP ( $\leq 500$ OPG), a leucocitose com neutrofilia e desvio à esquerda ocorreu em oito animais $(14,03 \%)$, dos quais quatro animais deste grupo $(7,01 \%)$ apresentaram uma eosinofilia associada. Nos asininos com CP (>500 OPG) foi predominante a leucocitose, com desvio à esquerda de neutrófilos associada a linfocitose ocorrendo em $(13,51 \%)$ dos animais, no entanto, aqueles com CP ( $\leq 500$ OPG) que apresentaram um quadro de leucocitose, com desvio à esquerda de neutrófilos, associada a eosinofilia e linfocitose foi de $(27,02 \%)$.

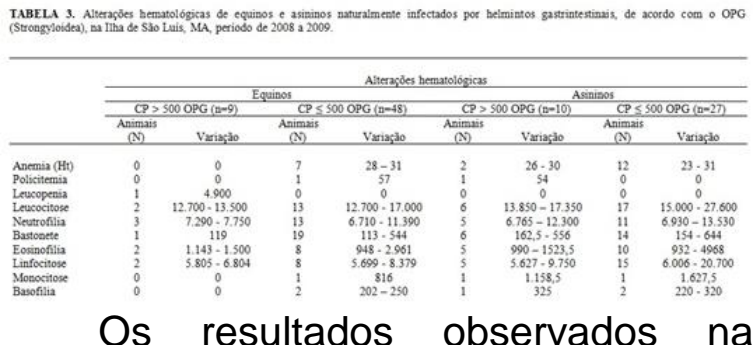

Tabela 4 apresentaram diferença estatística significativa entre os equinos com CP (>500 OPG) e valores médios encontrados de leucócitos totais de 
$9.855,55 \pm 3.147,26 / \mu \mathrm{L} ; \quad$ e, também quando comparado aos encontrados nos asininos com CP ( $\leq 500$ OPG) e média dos leucócitos totais de $14.998,15 \pm 6.029,88 / \mu \mathrm{L} \quad(\mathrm{P}<0,05)$. Ademais, ressalta-se que nos equinos, os helmintos com maior prevalência foram das espécies de $S$. vulgaris e $S$. edentatus, correspondendo associação destes de $72 \%$ de LPG (11.938 larvas/g de fezes). Enquanto, nos asininos foram aqueles pertencentes à Subfamília de Cyathostominae e $S$. vulgaris, com associação de 80\% LPG (2.840 larvas/g de fezes).

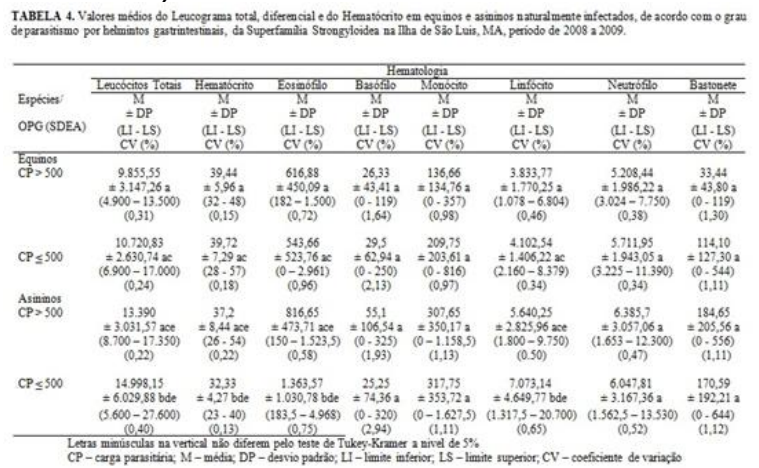

Em equinos apesar da associação do parasitismo entre o $S$. vulgaris e $S$. edentatus ter sido mais frequente em animais com CP (>500 OPG) ocorreu resposta leucocitária total discreta, acima do limite superior de referência adotado (12.500 leucócitos $/ \mu \mathrm{L}$ ), pois os valores médios de leucócitos totais corresponderam a 9.855,55 \pm $3.147,26 / \mu \mathrm{L}$, estando no limiar dos valores de referência para a espécie.

Não houve diferença estatística significativa tanto dentro dos grupos de equinos, como de asininos, para nenhuma variável analisada de leucócitos totais, diferenciais, e hematócrito $(P>0,05)$. Para as variáveis de leucócitos totais, hematócrito, eosinófilos e linfócitos comparando os asininos com CP ( $\leq 500$ OPG) houve diferença estatística significativa em relação ao grupo dos equinos com $\mathrm{CP}$ $(\leq 500$ OPG) e CP (>500 OPG) e asininos com CP $(>500$ OPG) $(P<0,05)$.
Os asininos com CP ( $\leq 500$ OPG) apresentaram valores médios de leucócitos totais de 14.998,15 $\pm 6.029,88 / \mu \mathrm{L} ;$ do hematócrito $32,33 \%$ $\pm 4,27$; de eosinófilos $1.363,57$ $\pm 1.030,78 / \mu \mathrm{L}$ e de linfócitos $7.073,14$ $\pm 4.649,77 / \mu \mathrm{L}$. Esses asininos apresentavam média de 131,48 \pm 127,96 OPG e $80 \%$ LPG (2.840 larvas/g de fezes), correspondente a associação entre os Cyathostominae (65\%) e $S$. vulgaris (15\%), demonstrados na Tabela 4, OPG na Tabela 1, LPG percentual e absoluto na Tabela 2).

Dessa forma foi observado que o grau de parasitismo não é a variável mais importante para determinar marcantes alterações hematológicas como a leucocitose associada à eosinofilia e/ou linfocitose. Os asininos do grupo de CP ( $\leq 500$ OPG) e do grupo de CP (>500 OPG) apresentaram médias elevadas de leucócitos totais, e ambos apresentavam infecção parasitária com percentual igual ou superior a $80 \%$ por Cyathostominae e $S$. vulgaris. Com base nesses valores demonstra-se que a associação Cyathostominae e $S$. vulgaris em asininos pode desencadear intensa resposta leucocitária independente do grau de parasitismo, sendo necessário o diagnóstico diferencial com outros agentes, verificado nos valores de OPG e também da ausência de evidências clínicas, inferi-se assim a natureza subclínica da infecção nesses animais. Além de demonstrar que para efeitos clínicos o OPG não é isoladamente um parâmetro fiel da carga parasitária em relação ao número de parasitas na forma adulta no hospedeiro segundo Uhlinger (1993), visto que ocorreram alterações hematológicas (baixo hematócrito, leucocitose associada a eosinofilia e, ou linfocitose) comprovadas neste trabalho através do teste estatístico. Assim os asininos do grupo de CP ( $\leq 500$ OPG) apresentaram valores médios de eosinófilos elevados 

aglomeração urbana, São Luís, Maranhão

entre e dentro dos grupos de animais estudados, constatando-se que a eosinofilia pode ser um sinalizador para o parasitismo nessa espécie, pois foi elevada a frequência da associação de larvas de Cyathostominae e $S$. vulgaris dentro desse grupo.

Nas infecções naturais por pequenos estrôngilos, foi relatada leucocitose, onde a ocorrência de anemia e eosinofilia dependem da intensidade e do tipo do quadro clínico apresentado (Giles et al. 1985, Reinemeyer 1986).

Alterações hematológicas como anemia e leucocitose associadas ou não à eosinofilia têm sido relatadas como consequência do parasitismo intestinal por estrongilídeos induzido experimentalmente em equinos (Amborski et al. 1974, Patton \& Drudge 1977, Klei et al. 1982, Dennis et al. 1992).

Segundo Reichmann et al. (2001) trabalhando com cavalos encontrou (OPG) com uma variação de 350 a 6.500 OPG (Md = 1.425 OPG) caracterizando um grau considerável de infecção por formas adultas de estrongilídeos, no qual encontraram quadro hematológico representado pelos valores médios de: hematócrito: $30,98 \pm 4,08 \%$; leucócitos totais: $12.495,83 \pm 2.776,26 / \mu \mathrm{L} ; \quad$ bastonetes: $28,98 \pm 114,43 / \mu \mathrm{L}$; neutrófilos: $7.970,77$ $\pm 2.534,33 / \mu \mathrm{L} ; \quad$ linfócitos: $3.921,62$ $\pm 1.585,29 / \mu \mathrm{L} ; \quad$ monócitos: 128,60 $\pm 187,10 / \mu \mathrm{L}$ e eosinófilos: 434,33 $\pm 398,07 / \mu \mathrm{L}$. Tais achados indicam a existência de leve leucocitose por neutrofilia, assim como uma tendência do quadro eritrocitário em se manter próximo aos limites inferiores de normalidade considerados para a espécie. Não foi observada, qualquer alteração quanto aos valores de eosinófilos, contradizendo em parte 0 conceito de que a eosinofilia deva acompanhar os quadros de parasitismo intestinal na espécie equina. Estes dados diferem dos resultados encontrados nessa pesquisa, com relação aos valores dos eosinófilos para o grupo dos asininos com CP $(\leq 500$ OPG), cujos valores encontrados foram bem superiores, e para os bastonetes nos equinos do grupo CP ( $\leq 500$ OPG) e asininos do grupo CP (>500 e $\leq 500$ OPG), porém se assemelham aos valores médios dos neutrófilos.

Tal contradição em relação aos resultados de Reichmann et al. (2001) pode ser explicada pelo fato da eosinofilia ocorrer particularmente como uma consequência da migração dos parasitos em fase larval após infecções, principalmente por $S$. vulgaris, e que no caso das infecções naturais, mistas por excelência, os Ciatostomíneos representam a grande maioria dos parasitos presentes no trato intestinal, quando comparados aos grandes estrôngilos. Esta afirmativa do autor diverge dos resultados desta pesquisa, pois os animais da espécie asinina apresentaram valores médios elevados para eosinófilos, sobretudo os do grupo com CP ( $\leq 500$ OPG).

Hubert et al. (2004) avaliando os sinais hematológicos em pôneis parasitados por $S$. vulgaris, observaram diferença significativa entre o grupo de animais parasitados em relação ao grupo controle, onde foram observados aumentos significativos na contagem total de glóbulos brancos, fibrinogênio, e concentração de proteínas plasmáticas. Além de diminuições significativas nas contagens de glóbulos vermelhos e do volume globular. Os resultados desta pesquisa concordam com o autor para o parasitismo por $S$. vulgaris, entretanto a existência da associação de Cyathostominae e $S$. vulgaris também determinou alterações leucocitárias, mesmo em decorrência de OPG com valores baixos em asininos, observados através do LPG. 


\section{CONCLUSÃO}

Os equinos $e$ asininos na população estudada da ilha de São Luís, Maranhão são naturalmente parasitados por helmintos gastrintestinais pertencentes à Subfamília Cyathostominae, e gênero/espécie Strongylus vulgaris, Strongylus edentatus. A associação de parasitismo entre Cyathostominae e Strongylus vulgaris apresenta valores médios elevados na contagem total de leucócitos, e absoluta de eosinófilos, principalmente nos asininos com carga parasitária ( $\leq 500 \mathrm{OPG})$, sinalizando que a eosinofilia é um parâmetro de infecção parasitária para a espécie.

O hematócrito apresenta tendência de aproximar-se dos valores de limites inferiores, porém a anemia não foi um achado clínico consistente com o grau de parasitismo para ambas as espécies equina e asinina.

O OPG é um indicador da condição parasitária do animal que pode ou não estar associado a alterações sanguíneas, visto que, os asininos com CP ( $\leq 500$ OPG) apresentam maior contagem total de leucócitos e eosinófilos em relação aos equinos com CP (>500 e $\leq 500$ OPG) evidenciando neste grupo de asininos, a rusticidade desta espécie animal.

Os asininos com CP $(>500$ e $\leq 500$ OPG) apresentam alterações hematológicas superiores aos valores de referência para os leucócitos totais (leucocitose), contagem de linfócitos (linfocitose) e bastonetes (caracterizando desvio à esquerda em ambos os grupos) e contagem de eosinófilos (eosinofilia) para os asininos com CP ( $\leq 500)$ quando comparados aos equinos estudados.

\section{REFERÊNCIAS}

AMBORSKI ,G.F.; BELLO, T.R.; TORBERT, B.J. Host response to experimentally induced infections of Strongylus vulgaris in parasite-free and naturally infected ponies. American Jr Vet Research, v. 35, p.1181-1188, 1974.

BARBOSA, O.F.; ROCHA, U.F.; SILVA, G.S. DA. et al. Nematóides Ciatostomíneos (Strongylidea, Strongylidae) em eqüinos criados a pasto no Estado de São Paulo, Brasil.

Semina, Ciências Agrárias, v.22, n.1, p.21-26, 2001.

CARVALHO, L.M.M.; GOMES, L.; CERNEA, M. et al. Parasitismo gastrintestinal e seu controlo em asininos e híbridos estabulados. Revista Portuguesa Ciências. Veterinárias, v.102, p.563-564 e 225-231, 2007.

COLLES, E.H. Patologia Clinica Veterinária. Manole, São Paulo. 1984. 556p.

DENNIS, V.A.; KLEI, R.T.; MILLER, A.M. et al. Immune response of pony foals during repeated infections of Strongylus vulgaris and regular ivermectin treatments. Veterinary Parasitology, v.42, p. 83-99, 1992.

DRUDGE, J.H.; LYONS, E.T.; SZANTO, J. Pathogenesis of migrating stages of helminths, with special reference to Strongylus vulgaris, p.199-214, 1966. In: Soulsby E.J.L. (Ed.),

Biology of Parasites: Emphasis on diagnosis, chemotherapy, and prophylaxis. Academic Press, New York.

DRUDGE, J.H. Clinical aspects of Strongylus vulgaris infection in the horse: Emphasis on diagnosis, chemotherapy, and prophylaxis.

Veterinary Clinical. North American., Large Animals. Practice, v.1, p.251-265, 1979.

DUNCAN, J.L.; PIRIE, H.M. The pathogenesis of single experimental infections with Strongylus vulgaris in foals. Research Veterinary Science, v.18, p.82-93, 1975.

GETACHE,W .M.; TRAWFORD, A.; FESEHA, G. et al. Gastrointestinal parasites of working donkeys of Ethiopia. Tropical Animal Health and Production, v.42, n.1, p.27-33, 2010.

GILES, J.L.; URQUHART, K.A.; LONGSTAFFE, J.A. Larval cyathostomiasis (immature trichonema-induced enteropathy): A report of 15 clinical cases. Equine Veterinary Journal, v.17, n.3, p.196-201, 1985.

GORDON, H.M.; WHITLOCK, H.V. A new technique for counting nematode eggs in sheep faeces. J. Commonwealth Science and Industrial Org., v.12, n.1, p.50-52, 1939. 
HOFFMANN, R.P. Diagnóstico de Parasitismo Veterinário. Sulina, Porto Alegre. 1987, 156p.

HUBERT, J.D.; SEAHORN, T.L.; KLEI, T.R. et al. Clinical signs and hematologic, cytokine, and plasma nitric oxide alterations in response to Strongylus vulgaris infection in helminth-naïve ponies. Canine Journal Veterinary Research, v.68, n.1, p.93-200, 2004.

IBGE. Anuário Estatístico. Instituto Brasileiro de Geografia e Estatística, RJ, 2002.

JAIN, N.C. Schalm's Vet. Hematology. 4th ed. Lea and Febiger, Philadelphia. 1986, 1221p.

JAIN, N.C. Essentials of Vet. Hematology. Lea and Febiger, Philadelphia. 1993, 417 p.

KLEI T,.R.; TORBERT, B.J.; OCHOA, R. et al. Morphologic and clinicopathologic changes following Strongylus vulgaris infections of immune and non-immune ponies. American Journal Vet. Res., v.43, n., p.1300-1307, 1982.

KLEI, T.R.; TURK, M.A.; MCCLURE, J.R. et al. Effects of repeated Strongylus vulgaris inoculations and concurrent ivermectin treatments on mesenteric arterial lesions in pony foals. American Journal Veterinary Research, v.51, p.654-660, 1990.

LICHTENFELS, J.R. Helminths of Domestic Equids: Illustrated keys to genera and species with emphasis on North American forms. Society Washington, Lawrence, v.42, p.1-92, 1975.

LIND, E.O.; EYSKER, M.; NILSSON, O. et al. Expulsion of small strongyle nematodes (Cyathostomin spp) following deworming of horses on a stud farm in Sweden. Veterinary Parasitology, v.115, n.4, p.289-299, 2003.

MEYER, D.J.; HARVEY, J.W. Veterinary Laboratory Medicine: Interpretation and diagnosis. 2nd ed. W.B. Saunders, Philadelphia. 2004, 351p.

MOLENTO, M.B. Resistência parasitária em helmintos de eqüídeos e propostas de manejo. Ciência Rural, v.35, n.6, p.1469-1477, 2005.

MOORE, R.M.; MUIR, W.W.; CAWRSE, M. et al. Systemic and colonic venous plasma eicosanoid and endotoxin concentrations, and colonic venous serum tumor necrosis factor and interleukin- 6 activities in horses during low-flow ischemia and reperfusion of the large colon.
American Journal Veterinary Research, v.56, n.8, p.656-663, 1995.

MORRIS, D.D. Endotoxemia in horses: A review of cellular and humoral mediators involved in its pathogenesis. Journal Veterinary

International Medicine, v.5, p.167-181, 1991.

\section{NUGEL/LABGEL. Núcleo de}

Geoprocessamento. Laboratório de Geopro., Universidade Estadual do Maranhão, 2008.

PATTON, S.; DRUDGE, J.H. Clinical response of pony foals experimentally infected with Strongylus vulgaris. American Journal Vet. Research, v.38, n.12, p.2059-2066, 1977.

PEREIRA, J.R.; VIANNA, S.S.S. Gastrointestinal parasitic worms in equines in the Paraíba Valley, State of São Paulo, Brazil. Veterinary

Parasitology, v.140, n.3, p.289-295, 2006.

REICHMANN, P.; LISBOA, J.A.N.; BALARIN, M.R.S. et al. Hematologic parameters in horses naturally infected with Strongyles. Semina, Ciências Agrárias, v.22, n.2, p.179-181, 2001.

REINEMEYER, C.R. Small strongyles: Recent advances. Veterinary Clinical North American, Equine Practice, v.2, n.2, p.281-312, 1986.

ROBERTS, F.H.S.; O'SULLIVAN, J.P. Methods for egg counts and larval cultures for strongyles infesting the gastrointestinal tract of cattle.

Australian Jr Agric Res, v.1, p.99-102, 1950.

SERRA-FREIRE, N.M. Planejamento em análise de pesquisas parasitológicas. Universi. Fed. Fluminense, Niterói. 2002, 199p.

SLOCOMB, J.O.D.1985. Pathogenesis of helminths in equines. Veterinary Parasitology, v.18, n.2, p.139-153, 1985.

UHLINGER ,C.A. Uses of faecal egg count data in equine practice. Compendium Contined Education Pract Vet., v.15, n.5, p.42-748, 1993.

UMUR, S.; AÇICI, M. A survey on helminth infections of equines in the Central Black Sea region, Turkey Turk. Journal Veterinary Animal Science, v.33, n.5, p.373-378, 2009.

USLU, U.; GUÇLU, F. Prevalence of endoparasites in horses and donkeys in turkey. Bull. Vet. Inst. Pulawy, v.51, p.237-240, 2007. 